\title{
Multifunctional Pharmaceutical Effects of the Antibiotic Daptomycin
}

\author{
Yang Ye $\mathbb{D}^{1},{ }^{1}$ Zijing Xia, ${ }^{2}$ Dan Zhang, ${ }_{1}^{1}$ Zenghua Sheng, ${ }^{1}$ Peng Zhang $\mathbb{D},{ }^{3}$ Hongxia Zhu, \\ Ningzhi Xu, ${ }^{1,4}$ and Shufang Liang $\mathbb{D}^{1}$ \\ ${ }^{1}$ State Key Laboratory of Biotherapy and Cancer Center, West China Hospital, Sichuan University and \\ Collaborative Innovation Center for Biotherapy, No.17, 3rd Section of People's South Road, Chengdu 610041, China \\ ${ }^{2}$ Department of Nephrology, West China Hospital, Sichuan University, Chengdu, Sichuan 610041, China \\ ${ }^{3}$ Department of Urinary Surgery, West China Hospital, Sichuan University, Chengdu, Sichuan 610041, China \\ ${ }^{4}$ Laboratory of Cell and Molecular Biology \& State Key Laboratory of Molecular Oncology, Cancer Institute \& Cancer Hospital, \\ Chinese Academy of Medical Sciences, Beijing 100034, China \\ Correspondence should be addressed to Shufang Liang; zizi2006@scu.edu.cn
}

Received 21 January 2019; Revised 24 April 2019; Accepted 14 May 2019; Published 27 May 2019

Academic Editor: Oguz R. Sipahi

Copyright (C) 2019 Yang Ye et al. This is an open access article distributed under the Creative Commons Attribution License, which permits unrestricted use, distribution, and reproduction in any medium, provided the original work is properly cited.

\begin{abstract}
Daptomycin (DAP), a cyclic lipopeptide produced by Streptomyces roseosporus, is a novel antibiotic to clinically treat various Gram-positive pathogenic bacteria-induced infections. Although DAP has a strong broad-spectrum bactericidal effect, recently rare bacterial antibiotic resistance against DAP gradually arises. The review is to summarize the normal indications of DAP, its off-label usage against several clinical pathogen infections, the unique antibacterial mechanisms of DAP, and the combination of antibiotic therapies for highly DAP-resistant pathogens. More noticeably, rising evidences demonstrate that DAP has new potential activity of anticancer and immunomodulatory effects. So far the multifunctional pharmaceutical effects of DAP deserve to be further explored for future clinical applications.
\end{abstract}

\section{Introduction}

Streptomyces are a rich source of bioactive secondary metabolites including numerous antibiotics. Among many antibacterial antibiotics, daptomycin (DAP), which is a secondary metabolite produced by Streptomyces roseosporus, is the first novel cyclic lipopeptide antibiotic (commercial name Cubicin $^{\circledR}$ ) approved clinically against various Gram-positive bacteria-induced infections [1].

DAP is an amphiphilic peptide with the molecular formula of $\mathrm{C}_{72} \mathrm{H}_{101} \mathrm{~N}_{17} \mathrm{O}_{26}$, which is composed of 13 amino acids and a decanoyl fatty acid tail [2]. In particular DAP contains both hydrophilic and hydrophobic amino acids, which determine its amphiphilic characteristic. One special amino acid, kynurenine, is highly fluorescent, and its intrinsic fluorescence is available to detect the interaction of DAP with cell membranes by fluorescence confocal microscopy.
DAP has a strong kill activity against most of Grampositive pathogenic bacteria. Because it is superior to vancomycin on the antimicrobial efficacy almost in all in vivo studies, DAP is commonly used as an effective therapy after vancomycin failure for the treatment of methicillinresistant Staphylococcus aureus (MRSA) infections [3]. Beside its effective antimicrobial activity, latest study suggests that DAP is a new potential therapeutic with anticancer and immunomodulatory activity.

The aim of this review is to summarize the normal indications and off-label usage of DAP, its unique antibacterial mechanisms, the combination antibiotic therapies, and its treatment for DAP-nonsusceptible (DNS) pathogens. Further, the options to overcome drug-resistance are also discussed. It is specially noticed that DAP has great potential as a new therapeutic against cancer and other immune relative diseases. 


\section{Broad Antimicrobial Activity of DAP}

2.1. Normal Indications of DAP. DAP (commercial name Cubicin ${ }^{\circledR}$ ) is originally approved by Food and Drug Administration (FDA) for the main indications including complicated skin and skin structure infections in adult and pediatric patients, Staphylococcus aureus (S. aureus) bloodstream infections (bacteremia), and those with right-sided infective endocarditis in adult patients [2]. And, compared with linezolid and vancomycin, DAP is well tolerated and has been proven to be a successful and less toxic alternative to vancomycin for the treatment of drug-resistant Gram-positive pathogens [2]. Despite sporadic reports of DAP resistance emerge in recent years, DAP is still a very important antimicrobial for current clinical practice around the world.

Moreover, DAP has been enlarged to be applied for various bacterial infections, including off-label use for osteomyelitis, meningitis, febrile neutropenia, and left-sided endocarditis.

2.2. Off-Label Usage of DAP. DAP shows good efficacy in antibiotic-resistant strains including MRSA and vancomycinresistant Enterococci (VRE), and DAP has been a preferred treatment strategy for VRE infections [4]. Furthermore, high dose of DAP is one alternative treatment recipe for penicillinresistant and cephalosporin-resistant pneumococcal meningitis and MRSA osteomyelitis [5, 6]. In addition, DAP has rapid bactericidal effects on Bacillus anthracis by reducing membrane potential so that it can be used as an alternate therapy for Bacillus anthracis infections [7]. Generally, DAP is a valuable treatment method in the management of other Gram-positive and difficult-to-treat infections, including meningitis, osteomyelitis, febrile neutropenia, and left-sided endocarditis.

2.2.1. Osteomyelitis. DAP is effective and safe in treatment for osteomyelitis [6]. Though it is not approved by FDA, the Infectious Diseases of Society of America guidelines for management of osteomyelitis recommend DAP as an alternative treatment method for osteomyelitis [8].

As DAP penetrates into synovial fluid and cancellous bone well, DAP concentrations in bone tissues are above the minimal inhibitory concentration (MIC) of DAP for S. aureus after a single-dose intravenous infusion [9]. Approximately $75 \%$ of cure rate is derived from the analysis of European Cubicin ${ }^{\circledR}$ Outcomes Registry and Experience registry data, with a low incidence of adverse events and drug resistance [10]. The optimal DAP dose to treat osteomyelitis has not been determined, although a study shows more than $6 \mathrm{mg} / \mathrm{kg}$ once daily is advantageous to compensate for low vascularization of bone tissue [8]. A high dose of $8-10 \mathrm{mg} / \mathrm{kg}$ DAP each day as monotherapy or in combination therapy with rifampin or trimethoprim-sulfamethoxazole optimizes its activity and avoids emergence of resistance [11].

2.2.2. Meningitis. At present, DAP is not indicated for meningitis treatment as it does not easily enter the central nervous compartments [12]. But DAP concentration in the cerebrospinal fluid is as higher as more than 100 times of the MIC [5]. Moreover, due to its excellent bactericidal response with no lysis, DAP is applicable for useful alternative treatment with penicillin-resistant and cephalosporin-resistant pneumococcal meningitis at high doses like $25 \mathrm{mg} / \mathrm{kg}$ [5]. So far, the experience with DAP for meningitis treatment mostly is confined to case reports and needs a large number of clinical evaluations [13].

2.2.3. Febrile Neutropenia. A few case reports have documented the success of DAP for Gram-positive infections in neutropenic patients $[14,15]$. DAP appears to be well tolerated and successfully control superinfections of bacteremia without marrow toxicity and complications in the neutropenic population [16]. Notably, the potential role of DAP in febrile neutropenia is yet to be determined, and clinical investigations are ongoing including the neutrophil recovery, blood culture results, and inflammatory condition of the entry sites.

2.2.4. Left-Sided Endocarditis. DAP was approved by FDA for the treatment of right-sided endocarditis in 2007, but its use for left-sided endocarditis therapy remains unapproved till now. Preliminary observation demonstrates good efficacy of DAP treatment in a small number of cases of left-sided infective endocarditis and MRSA-related left-sided endocarditis, where vancomycin is not effective $[17,18]$. But a wider series of patients with left heart endocarditis are now recruited to confirm the efficiency of DAP therapy [19].

In addition, DAP is not indicated for use against pneumonia and respiratory disease because it is inhibited by pulmonary surfactant, although DAP shows good curative effect in a mouse model with hematogenous pulmonary infection induced by MRSA [20].

\section{Antibacterial Mechanism of DAP}

The bactericidal mechanism of DAP is different from other traditional antibiotics [21], such as linezolid, vancomycin, and dalbavancin, so there is no cross-resistance between DAP and these antibiotics. To date, several molecular mechanism models of DAP have not completely elucidated its authenticity.

The majority of studies support that DAP acts on cell membranes in the presence of calcium ions, substituting $\mathrm{Ca}^{2+}$ with any of these divalent cations such as $\mathrm{Mn}^{2+}, \mathrm{Mg}^{2+}$, $\mathrm{Cu}^{2+}$, and $\mathrm{Ni}^{2+}$, causing a minimum 32 -fold increase in the MIC [22]. Once a 2:3 stoichiometric ratio of $\mathrm{Ca}^{2+}$ to DAP is reached, the conformation of DAP changes, which stimulates DAP oligomerization; finally its lipophilic tail inserts into bacterial cell membranes to penetrating membrane to induce loss of membrane potential and cell death [21, 23]. Our previous study has confirmed that the antibacterial mechanism of DAP is related to pathogen membrane proteins, including expression changes of multiple proteins [24]. The bacterial membrane potential is decreased and cell membrane is disrupted due to the upregulation of some proteins and downregulation of other proteins under DAP exposure, which contributes to rapid leakage of bacterial DNA and 
bactericidal effect [24]. While several pathogenic proteins are related to nucleic acid metabolism, DAP is probably involved in nucleic acid regulation. So the multitarget effects of DAP through proteomic dissection and validation are also worth considering $[24,25]$.

DAP seems to act on different targets for different infectious pathogens. DAP targets the fluid lipid domains, delocalizes different membrane proteins in Bacillus subtilis, and finally inhibits the synthesis of cell envelope by delocalizing different peripheral membrane proteins and suppressing the activity of biosynthetic enzymes [26]. DAP does not form discrete membrane pores in Bacillus subtilis $[26,27]$, which seems like self-contradictory with prevailing model about DAP ion channels. The differences in the fatty acyl composition of bacterial membranes could account for different susceptibilities toward membrane disruption [27]. The membrane pore formation is restricted by fatty acyl chain composition. Thus, the membrane pore is unable to form in DAP with cell membrane in Bacillus subtilis due to the bacterial membrane composition.

DAP exerts its antibacterial action not only on calcium ions but also with the presence of a high proportion of phosphatidylglycerol (PG) in membranes. The reduction of membrane lipids affects the affinity of DAP with membranes, which induces DAP inactivation by $S$. aureus [28]. Actually, DAP does not cross the giant unilamellar vesicles in the pathogen cell membranes [29, 30]. Generally, the antibacterial activity of DAP also depends on the membrane composition of various infectious bacteria.

\section{New Potential Pharmaceutical Effects of DAP}

4.1. DAP-Mediated Immunomodulatory Effect. Except own antimicrobial properties, DAP has been reported to exert other pharmaceutical effects, including immunomodulatory and anticancer activities (Figure 1).

In fact, lipopeptides interact with toll-like receptors to induce immune response [31]. DAP, a typical lipopeptide, may penetrate human monocytes to exert immunomodulatory effects $[32,33]$. DAP also regulates host immune response through changing cytokine production [33]. DAP may suppress cytokine expression in the case of host immune response stimulation against MRSA [34]. For instance, in the inflammatory response during sepsis, DAP treatment induces a significantly higher mRNA level of IL-6, IL-10, and IL-1 $\beta$ downregulation in lipopolysaccharide-activated monocytes. DAP also downregulates the gene expression of TLR1, TLR2, TLR6, and TLR9, whereas it makes an upregulation of TLR4 and TLR7 [35]. DAP treatment significantly induces lower TNF- $\alpha$ levels in cerebrospinal fluid of animal models compared with ceftriaxone [36].

Because the PG component in cell membrane is much richer in bacteria than that in eukaryotic cells, DAP has less effect on eukaryotic cells but its accumulation in alveoli will cause antigenic stimulation and continuous immune activation [37]. This is reason to explain the poor efficacy of DAP to treat left-sided infective endocarditis compared with right-sided infective endocarditis [38]. To date, the interaction of DAP with normal eukaryotic cell membrane constituents has little been reported.

DAP attributes largely to the reduced presentation of bacterial components to the host, so it is essential to explore the synergistic potential with some immunomodulators to indirectly modulate host immune function during bacterial infection. For example, DAP shows synergistic effects in combination with vitamin $\mathrm{E}$ [33]. The treatment with vitamin E prior to infection is effective for improving efficacy of DAP in MRSA-caused wound infection [39]. This better combination effects have been validated in mouse models, in which CD49b + cells are increased compared with single treatment with vitamin E or DAP alone [40]. Although the immunomodulatory mechanism of DAP is not completely explored, it is reasonable to predict DAP potential usage for immune-relative diseases. So DAP may have the potential to indirectly modulate host immune function during resolution of infection.

4.2. Antitumor Potential of DAP. Peptides have displayed anticancer properties [41]. Some marine cyclic peptides have anticancer applications, such as dolastatines and soblidotin, especially cyclic guineamides A-F which are similar to DAP in the molecular structures [41].

There are some preliminary investigations on DAP anticancer activities. DAP inhibits cell proliferation of cancer cells including MCF-7 and HCT116, and RPS19 is identified to be a biophysical and biologically related target protein of DAP through an unbiased reverse chemical proteomics screen [42]. With higher expression levels of RPS19 in certain colon cancer cells, RPS19 may be a promising drug target protein for DAP anticancer activity [42].

Gram-positive infections are a main cause of morbidity and mortality in cancer patients. Though clinical data for DAP in the treatment of cancer patients are limited, there are some evidences of DAP usage particularly in some special Gram-positive infections in cancer patients with neutropenia (Table 1) [43]. In addition, DAP is an attractive option for the treatment of Gram-positive catheter-related bloodstream infections in cancer patients [44] and implantable intraarterial catheters in liver metastases of colon cancer [45].

Compared to other antibiotics used in cancer infections, in terms of efficacy and drug resistance, DAP is a safe and effective therapy in the treatment of bacterial infections induced by Gram-positive pathogens [46]. Both vancomycin and linezolid have limitations with respect to their use in patients with neutropenia. Compared with vancomycin, the nephrotoxicity of DAP is not significant; cancer patients treated with DAP achieve earlier microbiological eradication [47]. Furthermore, unlike linezolid, DAP is more effective in eradicating catheter-related MRSA infections [48].

An interesting question is whether the combination of DAP and antitumor drugs (e.g., oxaliplatin and letrozole) can enhance the activity of anticancer drugs. Because of the low immunity of cancer patients, perhaps DAP has synergistic antitumor effects for cancer patients in combination with anticancer drugs. However, this conjecture needs further study. 
TABLE 1: DAP treatment in cancer.

\begin{tabular}{|c|c|c|c|c|}
\hline Cancer type & Infections type & Pathogen & Dosage & Ref \\
\hline $\begin{array}{l}\text { Hematologic malignancy } \\
\text { Solid tumor }\end{array}$ & $\begin{array}{l}\text { Catheter-related } \\
\text { blood stream infections }\end{array}$ & Gram-positive organisms & $6 \mathrm{mg} / \mathrm{kg} /$ day i.v. & [44] \\
\hline Colon cancer & $\begin{array}{l}\text { Implantable intra-arterial } \\
\text { catheter infections }\end{array}$ & Not mentioned & 10 mg/kg/day i.v. & {$[45]$} \\
\hline $\begin{array}{l}\text { Hematologic malignancy } \\
\text { Solid tumor }\end{array}$ & Bacteremia & $\begin{array}{l}\text { Vancomycin-resistant } \\
\text { Enterococcus }\end{array}$ & About $6.1 \mathrm{mg} / \mathrm{kg} /$ day i.v. & [46] \\
\hline Acute myeloid leukemia & Bacteremia & $\begin{array}{l}\text { Teicoplanin-resistant } \\
\text { Gram-positive cocci }\end{array}$ & $8 \mathrm{mg} / \mathrm{kg} /$ day i.v. & {$[16]$} \\
\hline
\end{tabular}

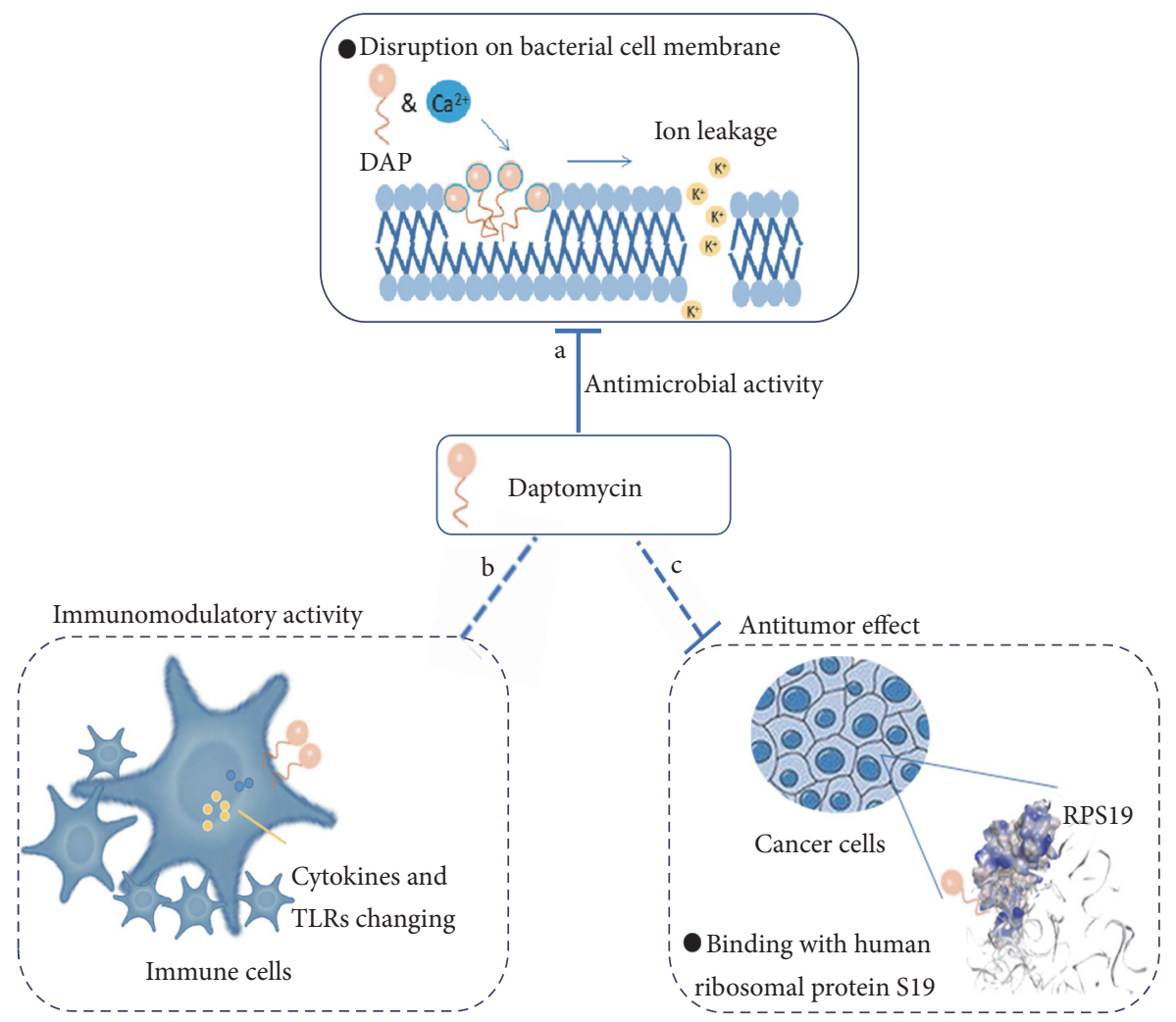

FIGURE 1: Multifunctional pharmaceutical effects of the antibiotic daptomycin. Besides antimicrobial properties, DAP is associated with anticancer and immunomodulatory activity.

\section{Overcoming Antibiotic Resistance}

Gene mutations, changes in surface charges of cell membranes, the thickening of bacterial cell walls, and the altered lipid metabolism are all associated with DAP resistance in Gram-positive pathogens, including Enterococcus faecalis [49], S. aureus [28], Streptococcus mitis/oralis [50], and Bacillus subtilis [51]. Although this resistance is very sparse without wide spreading, the occurrence of DAP resistance during therapy seems to be an important problem affecting the clinical efficacy of DAP [28]. New DAP-based antibiotic discovery and combination therapy are considered to prevent the emergence of highly drug-resistant pathogenic bacteria [52].

5.1. DAP-Derived Antibiotics. Cyclic peptide antibiotics have unconventional antibacterial mechanisms due to their unique structure. Novel cyclic peptides have been derived from a natural product, fusaricidin/LI-F, to have better antibacterial activity through screening a positional scanning combinatorial library [53]. And, with the increasing of the overall hydrophobicity and the net positive charge of cyclic lipopeptides, the antibacterial activity will be improved. Besides, bicyclic peptides may be a good way to discover new cyclic bioactive peptides [54]. So de novo rational design and biosynthesis are applicable to optimize and create better therapeutic polypeptide-like drugs [52]. But, inconsistently, DAP dimer, which is composed of two subunits, has a very low activity on vegetative $S$. aureus because the dimer does not act on cell membranes due to the bivalent aliphatic acyl chain between two subunits [55].

In addition, enhancing membrane-binding property is an option to optimize antibiotics. For instance, N-terminal or 
TABLE 2: DAP combination therapies against ${ }^{\mathrm{a}} \mathrm{DNS}$ strains.

\begin{tabular}{lccccc}
\hline DAP combination & Strains & $\begin{array}{c}\text { For DNS } \\
\text { strains }\end{array}$ & Mechanisms & Activity \\
\hline DAP+Oxacillin & MRSA & ${ }^{\mathrm{b} S y n}$ & $\begin{array}{c}\text { Changes in peptidoglycan insertion } \\
\text { Reduction in membrane amounts of }{ }^{\mathrm{C} P B P 2 a}\end{array}$ & In vivo, In vitro & [58] \\
DAP+Ceftaroline & MRSA & Syn & Enhance the binding of DAP & In vitro & [61, 62] \\
DAP+Gentamicin & MRSA & Syn & Not mentioned & In vitro & In vitro \\
DAP+Fosfomycin & VRE & Syn & Reduction in cell surface charge & In vitro \\
DAP+Ampicillin & VRE & Syn & Reduction in cell surface charge & [59] \\
DAP+Ceftriaxone & Streptococcus & Syn & Prevent DAP resistance emergence & In vitro & [63] \\
\hline
\end{tabular}

${ }^{\mathrm{a}}$ DNS, DAP-nonsusceptible.

${ }^{\mathrm{b}}$ Syn, synergy.

${ }^{\mathrm{c}} \mathrm{PBP} 2 \mathrm{a}$, penicillin binding protein $2 \mathrm{a}$.

C-terminal lipophilic groups (myristoyl, palmitoyl, geranyl, and farnesyl) of vancomycin are appending onto the Cterminus to enhance the membrane binding with antibiotic and drug concentration at the target site, which increases killing activity against MRSA and DAP-resistant bacteria [56]. The differences in the fatty acyl composition of bacterial membranes are related with DAP binding to the membranes. So, DAP activity is also influenced by the optimization of fatty acid side chains. For example, surotomycin, which is a DAP derivative, exhibits significant improvement of in vitro activity compared with DAP against Clostridium difficile [57]. The only change between them is the specific fatty acid side chains. Therefore, fatty acid side chains play an important role in the binding of antibiotics to cell membranes and affect antibacterial activity. More importantly, the optimal balance between strong antibacterial activity and optimum structure is essential for structure modification.

5.2. Synergism. A combination therapy of two antibiotics is recommended to prevent the emergency of DAP resistance. Herein, several combination antibiotic regimens are reported to be effective against DAP-resistant bacterial infections, and these combinations are summarized in Table 2.

5.2.1. DAP Combined with $\beta$-Lactams. DAP combined with $\beta$-lactams, especially with ceftaroline and ampicillin, has better killing of DAP nonsusceptibility in clinical MRSA and enterococci isolates [58-60]. The affinity of DAP and bacterial membranes is found to be enhanced by the addition of ampicillin, which is proven to be the synergy mechanism between ampicillin and DAP in enterococci [59]. A combination of DAP and ceftaroline is active against DNS MRSA and Streptococcus mitis in vitro by enhancing the binding of DAP and cell membranes [61-63]. Interestingly, S. aureus inactivates DAP through releasing membrane phospholipids, which can be inhibited with the presence of $\beta$-lactam antibiotic [28]. DAPoxacillin exposure reduces membrane amounts of penicillinbinding protein 2 and redirects the localization, which induces cell wall perturbations and increases susceptibility to oxacillin [58]. (a)

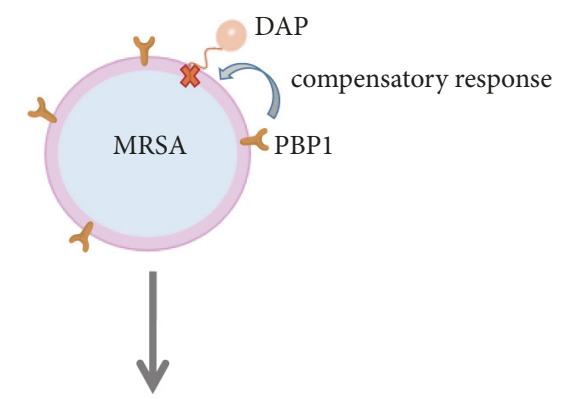

(b)

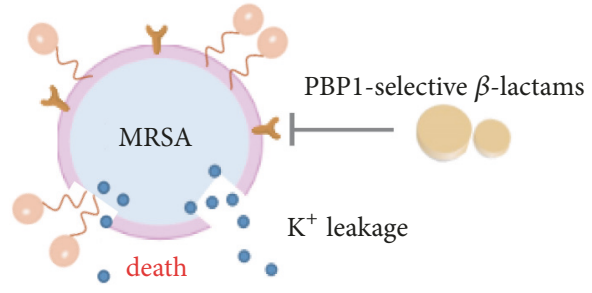

FIGURE 2: The action mechanism of DAP combined with $\beta$-lactams. (a) Penicillin binding protein 1 (PBP1) activity is related to DAPinduced metabolic stress, and it forms an adaptive response to DAPmediated surface injury. (b) Targeting inactivation of PBP1 makes DAP-nonsusceptible $S$. aureus strains revert to subsequent DAPsusceptible strains, which enhances the binding of DAP with cell membrane and then induces cell death.

$\mathrm{DAP} / \beta$-lactams have intracellular activity against MSSA and MRSA. Oxacillin binds to penicillin-binding proteins, which are enzymes involved in the synthesis of peptidoglycan. So oxacillin enhances the activity of DAP due to suppression of cell wall synthesis [64]. Meanwhile, penicillin binding protein 1 (PBP1) activity is associated with DAPinduced metabolic stress. PBP1 is one of penicillin binding proteins in S. aureus, which is a target of $\beta$-lactam. PBP1 is a crucial compensatory response to DAP injury. Targeting inactivation of PBP1 induces DNS S. aureus reversion to a subsequent DAP-susceptible strain, which alters the efficacy of DAP killing. Therefore DAP combined with PBP1-selective $\beta$-lactams may be a suitable strategy (Figure 2) [65]. In short, the synergistic bactericidal effects of DAP with other chemicals on the DNS strains are mostly dependent with 
TABLE 3: Novel DAP conjugates.

\begin{tabular}{|c|c|c|c|c|c|}
\hline DAP conjugates & The compounds coupled with DAP & Strains & Mechanisms & Activity & Refs \\
\hline $\begin{array}{l}\text { Synthetic } \\
\text { sideromycin }\end{array}$ & $\begin{array}{l}\text { A mixed ligand analog of the natural } \\
\text { A. baumannii selective siderophore }\end{array}$ & A. baumannii & $\begin{array}{l}\text { Actively transport DAP into } \\
\text { Gram-negative bacteria }\end{array}$ & In vitro, In vivo & {$[71]$} \\
\hline Dapt-PEG-DSPE & $\begin{array}{l}\text { N-hydroxysuccinimidyl-polyethylene } \\
\text { glycol-1,2-distearoyl-sn-glycero-3- } \\
\text { phosphoethanolamine }\end{array}$ & MRSA252 & $\begin{array}{l}\text { Target on the surface of } \\
\text { liposomes }\end{array}$ & In vitro, In vivo & {$[73]$} \\
\hline $\begin{array}{l}\text { AgNCs-DAP } \\
\text { hybrid }\end{array}$ & Silver nanoclusters (AgNCs) & $\begin{array}{c}\text { S. aureus } \\
\text { ATCC } 25923\end{array}$ & $\begin{array}{c}\text { Generate localized high ROS } \\
\text { concentration } \\
\text { Damage the bacterial } \\
\text { membrane and DNA }\end{array}$ & In vitro & {$[74]$} \\
\hline
\end{tabular}

the synergistic drug influence on membrane proteins, by facilitating an increase of DAP binding and rapidly leading to cell death.

5.2.2. DAP Combined with Other Antibiotics. There are attempts for the synergy with DAP and other antibiotics, such as gentamicin [66], dalbavancin [67], and fosfomycin [68]. And the MIC values of DAP in combination with these antibiotics are lower than each alone [67, 68].

Fosfomycin is a phosphonic acid derivative, which is active against both Gram-negative and Gram-positive bacteria by inhibiting peptidoglycan synthesis. DAP influences peptidoglycan synthesis based on the above mechanisms. So fosfomycin can increase the activity of DAP against VRE through modulating cell surface charge, which indicates that the mechanism is similar to the combination of $\beta$-lactams and DAP [69]. Similarly, the combination of DAP plus gentamycin has demonstrated better activity against MRSA and also provides a promising synergy required to overcome DAP resistance in complicated infections [66]. Gentamicin is one of the most active aminoglycosides, which is used to treat bacterial infections, especially those caused by Gramnegative bacteria. It can bind to ribosomal 30s subunit and block bacterial protein synthesis, which is different from the action mechanism of DAP [66]. The synergistic mechanism is unclear, and the synergy is only demonstrated in in vitro model, so further study should focus on the synergy in vivo and clinical applications.

\section{Future Prospects}

Except for antimicrobial properties, DAP may have other pharmaceutical activities like immunomodulatory and antitumor effects. It still has a great development prospect to extend DAP clinical potentials. Firstly, extending the antimicrobial spectrum of DAP is pending because of the unapparent inhibition effect of DAP on Gram-negative bacteria. Recently, it has been reported that DAP has some influence on Gram-negative bacteria in combination with colistin [70]. Further, a novel DAP conjugate, which consists of DAP and a mixed ligand analog of the natural Acinetobacter baumannii (A.baumannii) selective siderophore, not only retains the inhibitory effect on Gram-positive bacteria but also has potent activity against multidrug-resistant strains of
A.baumannii [71]. Similarly, several DAP novel conjugates are summarized in Table 3.

There are few drug delivery research reports to improve DAP preparations. In the interest of increasing the effectiveness, DAP-loaded nanoparticles are used directly for treatment of endophthalmitis [72]. Additionally, DAP-modified liposomes exhibit highly specific targeting and therapeutic efficacy against MRSA [73]. More interestingly, an efficient hybrid of inorganic antimicrobial agent with DAP, which is conjugated through a strong covalent bond, enhances bactericidal activity by generating ROS, further damaging cell lipid membranes, and accelerating cell death [74]. In order to increase drug loading, some researches develop a positively charged proliposomal formulation for oral administration of DAP [75]. So, improving preparation of DAP, such as oral preparation, may be a good method to explore the potential of DAP.

\section{Abbreviations \\ DAP: Daptomycin \\ MRSA: Methicillin-resistant Staphylococcus aureus \\ VRE: Vancomycin-resistant Enterococci \\ FDA: Food and Drug Administration \\ MIC: Minimal inhibitory concentration \\ PG: Phosphatidylglycerol \\ PBP1: Penicillin binding protein 1 \\ DNS: DAP-nonsusceptible.}

\section{Conflicts of Interest}

The authors declare that they have no conflicts of interest.

\section{Acknowledgments}

This work was supported by the National 863 High Tech Foundation (2014AA020608), the Health Commission of Sichuan Province (17ZD045), and Chengdu Science and Technology Program (2017-GH02-00062-HZ).

\section{References}

[1] L. Robbel and M. A. Marahiel, "Daptomycin, a bacterial lipopeptide synthesized by a nonribosomal machinery," The 
Journal of Biological Chemistry, vol. 285, no. 36, pp. 2750127508, 2010.

[2] M. Heidary, A. D. Khosravi, S. Khoshnood, M. J. Nasiri, S. Soleimani, and M. Goudarzi, "Daptomycin," Journal of Antimicrobial Chemotherapy, vol. 73, no. 1, pp. 1-11, 2018.

[3] R. M. Humphries, S. Pollett, and G. Sakoulas, "A current perspective on daptomycin for the clinical microbiologist," Clinical Microbiology Reviews, vol. 26, no. 4, pp. 759-780, 2013.

[4] F. Foolad, B. D. Taylor, S. A. Shelburne, C. A. Arias, and S. L. Aitken, "Association of daptomycin dosing regimen and mortality in patients with VRE bacteraemia: a review," Journal of Antimicrobial Chemotherapy, vol. 73, no. 9, pp. 2277-2283, 2018.

[5] M. Vivas, E. Force, C. Garrigós et al., "Experimental study of the efficacy of daptomycin for the treatment of cephalosporinresistant pneumococcal meningitis," Journal of Antimicrobial Chemotherapy, vol. 69, no. 11, pp. 3020-3026, 2014.

[6] S. Hirsiger, İ. Ilgaz, and İ. Uçkay, "New antibiotics in the therapy of osteomyelitis," Mediterranean Journal of Infection Microbes and Antimicrobials, vol. 6, p. 15, 2017.

[7] Y.-H. Xing, W. Wang, S.-Q. Dai et al., "Daptomycin exerts rapid bactericidal activity against Bacillus anthracis without disrupting membrane integrity," Acta Pharmacologica Sinica, vol. 35, no. 2, pp. 211-218, 2014.

[8] K. Malizos, J. Sarma, R. A. Seaton et al., "Daptomycin for the treatment of osteomyelitis and orthopaedic device infections: real-world clinical experience from a European registry," European Journal of Clinical Microbiology \& Infectious Diseases, vol. 35, no. 1, pp. 111-118, 2016.

[9] D. Montange, F. Berthier, G. Leclerc et al., "Penetration of daptomycin into bone and synovial fluid in joint replacement," Antimicrobial Agents and Chemotherapy, vol. 58, no. 7, pp. 39913996, 2014.

[10] R. Andrew Seaton, K. N. Malizos, P. Viale et al., "Daptomycin use in patients with osteomyelitis: a preliminary report from the EU-CORESM database," Journal of Antimicrobial Chemotherapy, vol. 68, no. 7, pp. 1642-1649, 2013.

[11] L. M. Avery, M. E. Steed, A. E. Woodruff, M. Hasan, and M. J. Rybak, "Daptomycin-nonsusceptible vancomycinintermediate Staphylococcus aureus vertebral osteomyelitis cases complicated by bacteremia treated with high-dose daptomycin and trimethoprim-sulfamethoxazole," Antimicrobial Agents and Chemotherapy, vol. 56, no. 11, pp. 5990-5993, 2012.

[12] R. Nau, M. Djukic, A. Spreer, S. Ribes, and H. Eiffert, "Bacterial meningitis: an update of new treatment options," Expert Review of Anti-infective Therapy, vol. 13, no. 11, pp. 1401-1423, 2015.

[13] B. M. Knoll, M. Hellmann, and C. N. Kotton, "Vancomycinresistant Enterococcus faecium meningitis in adults: case series and review of the literature," Infectious Diseases, vol. 45, no. 2, pp. 131-139, 2013.

[14] J. S. Bubalo, R. Kullar, and R. T. Maziarz, "A pilot study of the efficacy and safety of empiric daptomycin therapy in oncology patients with fever and severe neutropenia," Therapeutic Advances in Infectious Disease, vol. 1, no. 6, pp. 183-190, 2013.

[15] O. R. Sipahi, H. Kahraman, H. A. Erdem et al., "Daptomycin vs. glycopeptides in the treatment of febrile neutropenia: results of the Izmir matched cohort study," Infection, vol. 47, no. 2, pp. 259-266, 2019.

[16] K. Matsuda, J. Koya, K. Toyama et al., "A therapeutic benefit of daptomycin against glycopeptide-resistant gram-positive cocci bloodstream infections under neutropenia," Journal of Infection and Chemotherapy, vol. 23, no. 11, pp. 788-790, 2017.
[17] P. Xu, H. Zeng, M. Zhou, J. Ouyang, B. Chen, and Q. Zhang, "Successful management of a complicated clinical crisis: a patient with left-sided endocarditis and secondary hemophagocytic lymphohistiocytosis: a rare case report and literature review," Medicine (United States), vol. 96, no. 51, article no. e9451, 2017.

[18] I. M. Gould, J. M. Miró, and M. J. Rybak, "Daptomycin: the role of high-dose and combination therapy for Gram-positive infections," International Journal of Antimicrobial Agents, vol. 42, no. 3, pp. 202-210, 2013.

[19] A. Guleri, R. Utili, P. Dohmen et al., "Daptomycin for the treatment of infective endocarditis: results from european cubicin ${ }^{\circledR}$ outcomes registry and experience (EU-CORE)," Infectious Diseases and Therapy, vol. 4, no. 3, pp. 283-296, 2015.

[20] Y. Harada, K. Yanagihara, K. Yamada et al., "In vivo efficacy of daptomycin against methicillin-resistant Staphylococcus aureus in a mouse model of hematogenous pulmonary infection," Antimicrobial Agents and Chemotherapy, vol. 57, no. 6, pp. 2841-2844, 2013.

[21] S. D. Taylor and M. Palmer, "The action mechanism of daptomycin," Bioorganic \& Medicinal Chemistry, vol. 24, no. 24, pp. 6253-6268, 2016.

[22] S. W. Ho, D. Jung, J. R. Calhoun et al., "Effect of divalent cations on the structure of the antibiotic daptomycin," European Biophysics Journal, vol. 37, no. 4, pp. 421-433, 2008.

[23] M.-T. Lee, W.-C. Hung, M.-H. Hsieh, H. Chen, Y.-Y. Chang, and H. W. Huang, "Molecular state of the membrane-active antibiotic daptomycin," Biophysical Journal, vol. 113, no. 1, pp. 82-90, 2017.

[24] W. Ma, D. Zhang, G. Li et al., "Antibacterial mechanism of daptomycin antibiotic against Staphylococcus aureus based on a quantitative bacterial proteome analysis," Journal of Proteomics, vol. 150, pp. 242-251, 2017.

[25] B. Chen, D. Zhang, X. Wang et al., "Proteomics progresses in microbial physiology and clinical antimicrobial therapy," European Journal of Clinical Microbiology \& Infectious Diseases, vol. 36, no. 3, pp. 403-413, 2017.

[26] A. Müller, M. Wenzel, H. Strahl et al., "Daptomycin inhibits cell envelope synthesis by interfering with fluid membrane microdomains," Proceedings of the National Acadamy of Sciences of the United States of America, vol. 113, no. 45, pp. E7077-E7086, 2016.

[27] R. Taylor, D. Beriashvili, S. Taylor, and M. Palmer, "Daptomycin pore formation is restricted by lipid acyl chain composition," ACS Infectious Diseases, vol. 3, no. 11, pp. 797-801, 2017.

[28] V. Pader, S. Hakim, K. L. Painter, S. Wigneshweraraj, T. B. Clarke, and A. M. Edwards, "Staphylococcus aureus inactivates daptomycin by releasing membrane phospholipids," Nature Microbiology, vol. 2, article no. 16194, 2016.

[29] M. A. Kreutzberger, A. Pokorny, and P. F. Almeida, "Daptomycin-phosphatidylglycerol domains in lipid membranes," Langmuir, vol. 33, no. 47, pp. 13669-13679, 2017.

[30] Y.-F. Chen, T.-L. Sun, Y. Sun, and H. W. Huang, "Interaction of daptomycin with lipid bilayers: a lipid extracting effect," Biochemistry, vol. 53, no. 33, pp. 5384-5392, 2014.

[31] I. W. Hamley, "Lipopeptides: from self-assembly to bioactivity," Chemical Communications, vol. 51, no. 41, pp. 8574-8583, 2015.

[32] A. L. Baltch, W. J. Ritz, L. H. Bopp, P. B. Michelsen, and R. P. Smith, "Antimicrobial activities of daptomycin, vancomycin, and oxacillin in human monocytes and of daptomycin in combination with gentamicin and/or rifampin in human monocytes 
and in broth against Staphylococcus aureus," Antimicrobial Agents and Chemotherapy, vol. 51, no. 4, pp. 1559-1562, 2007.

[33] T. Kelesidis, "The interplay between daptomycin and the immune system," Frontiers in Immunology, vol. 5, article no. 52, 2014.

[34] T. Tirilomis, "Daptomycin and its immunomodulatory effect: consequences for antibiotic treatment of methicillin-resistant Staphylococcus aureus wound infections after heart surgery," Frontiers in Immunology, vol. 5, p. 97, 2014.

[35] C. Bode, S. Muenster, B. Diedrich et al., "Linezolid, vancomycin and daptomycin modulate cytokine production, toll-like receptors and phagocytosis in a human in vitro model of sepsis," The Journal of Antibiotics, vol. 68, no. 8, pp. 485-490, 2015.

[36] M. Vivas, E. Force, C. El Haj, F. Tubau, J. Ariza, and C. Cabellos, "Experimental study of cerebrospinal fluid tumor necrosis factor-alpha release in penicillin- and cephalosporinresistant pneumococcal meningitis treated with different antibiotic schedules," Journal of Microbiology, Immunology and Infection, vol. 50, no. 4, pp. 435-439, 2017.

[37] J. A. Silverman, L. I. Mortin, A. D. G. VanPraagh, T. Li, and J. Alder, "Inhibition of daptomycin by pulmonary surfactant: In vitro modeling and clinical impact," The Journal of Infectious Diseases, vol. 191, no. 12, pp. 2149-2152, 2005.

[38] Y. Higashi, S. Nakamura, Y. Tsuji et al., "Daptomycin-induced eosinophilic pneumonia and a review of the published literature," Internal Medicine, vol. 57, no. 2, pp. 253-258, 2018.

[39] M. Provinciali, O. Cirioni, F. Orlando et al., "Vitamin E improves the in vivo efficacy of tigecycline and daptomycin in an animal model of wounds infected with meticillin-resistant Staphylococcus aureus," Journal of Medical Microbiology, vol. 60, no. 12, pp. 1806-1812, 2011.

[40] E. Pierpaoli, O. Cirioni, A. Barucca et al., "Vitamin E supplementation in old mice induces antimicrobial activity and improves the efficacy of daptomycin in an animal model of wounds infected with methicillin-resistant Staphylococcus aureus," Journal of Antimicrobial Chemotherapy, vol. 66, no. 9, pp. 2184-2185, 2011.

[41] Y. Lee, C. Phat, and S.-C. Hong, "Structural diversity of marine cyclic peptides and their molecular mechanisms for anticancer, antibacterial, antifungal, and other clinical applications," Рeptides, vol. 95, pp. 94-105, 2017.

[42] M. P. Gotsbacher, S. Cho, H. J. Kwon, and P. Karuso, "Daptomycin, a last-resort antibiotic, binds ribosomal protein S19 in humans," Proteome Science, vol. 15, p. 16, 2016.

[43] K. V. Rolston, S. A. McConnell, J. Brown, and K. C. Lamp, "Daptomycin use in patients with cancer and neutropenia: data from a retrospective registry," Clinical Advances in Hematology \& Oncology, vol. 8, no. 4, pp. 249-256, 2010.

[44] A.-M. Chaftari, R. Hachem, V. Mulanovich et al., "Efficacy and safety of daptomycin in the treatment of Gram-positive catheter-related bloodstream infections in cancer patients," International Journal of Antimicrobial Agents, vol. 36, no. 2, pp. 182-186, 2010.

[45] M.-A. Vandenhende, J. Buret, F. Camou, P. Morlat, and F. Bonnet, "Successful daptomycin lock therapy for implantable intraarterial catheter infection in a patient with liver metastases of colon cancer," Diagnostic Microbiology and Infectious Disease, vol. 78, no. 4, pp. 497-498, 2014.

[46] K. Patel, R. Kabir, S. Ahmad, and S. L. Allen, "Assessing outcomes of adult oncology patients treated with linezolid versus daptomycin for bacteremia due to vancomycin-resistant
Enterococcus," Journal of Oncology Pharmacy Practice, vol. 22, no. 2, pp. 212-218, 2016.

[47] K. V. I. Rolston, M. Kapadia, J. Tarrand, E. Coyle, and R. A. Prince, "Spectrum of Gram-positive bacteraemia and in vitro activities of daptomycin, linezolid and vancomycin against organisms isolated from cancer patients," International Journal of Antimicrobial Agents, vol. 41, no. 6, pp. 516-520, 2013.

[48] I. Raad, H. Hanna, Y. Jiang et al., "Comparative activities of daptomycin, linezolid, and tigecycline against catheterrelated methicillin-resistant Staphylococcus bacteremic isolates embedded in biofilm," Antimicrobial Agents and Chemotherapy, vol. 51, no. 5, pp. 1656-1660, 2007.

[49] E. V. K. Ledger, V. Pader, and A. M. Edwards, "Enterococcus faecalis and pathogenic streptococci inactivate daptomycin by releasing phospholipids," Microbiology (United Kingdom), vol. 163, no. 10, pp. 1502-1508, 2017.

[50] N. N. Mishra, T. T. Tran, R. Seepersaud et al., "Perturbations of phosphatidate cytidylyltransferase (CdsA) mediate daptomycin resistance in Streptococcus mitis/oralis by a novel mechanism," Antimicrobial Agents and Chemotherapy, vol. 61, no. 4, Article ID e02435-16, 2017.

[51] A.-B. Hachmann, E. Sevim, A. Gaballa, D. L. Popham, H. Antelmann, and J. D. Helmann, "Reduction in membrane phosphatidylglycerol content leads to daptomycin resistance in Bacillus subtilis," Antimicrobial Agents and Chemotherapy, vol. 55, no. 9, pp. 4326-4337, 2011.

[52] D. Zhang, Y. He, Y. Ye et al., "Little antimicrobial peptides with big therapeutic roles," Protein and Peptide Letters, vol. 26, pp. 1-14, 2019.

[53] N. Bionda, R. M. Fleeman, C. De La Fuente-Núñez et al., "Identification of novel cyclic lipopeptides from a positional scanning combinatorial library with enhanced antibacterial and antibiofilm activities," European Journal of Medicinal Chemistry, vol. 108, pp. 354-363, 2016.

[54] K. Deyle, X.-D. Kong, and C. Heinis, "Phage selection of cyclic peptides for application in research and drug development," Accounts of Chemical Research, vol. 50, no. 8, pp. 1866-1874, 2017.

[55] R. M. Taylor, B. Scott, S. Taylor, and M. Palmer, "An acyl-linked dimer of daptomycin is strongly inhibited by the bacterial cell wall," ACS Infectious Diseases, vol. 3, no. 7, pp. 462-466, 2017.

[56] M. A. Blaskovich, K. A. Hansford, Y. Gong et al., "Proteininspired antibiotics active against vancomycin- and daptomycin-resistant bacteria," Nature Communications, vol. 9, no. 1, p. 22, 2018.

[57] N. Yin, J. Li, Y. He et al., "Structure-activity relationship studies of a series of semisynthetic lipopeptides leading to the discovery of surotomycin, a novel cyclic lipopeptide being developed for the treatment of clostridium difficile -associated diarrhea," Journal of Medicinal Chemistry, vol. 58, no. 12, pp. 5137-5142, 2015.

[58] A. Renzoni, W. L. Kelley, R. R. Rosato et al., "Molecular bases determining daptomycin resistance-mediated resensitization to $\beta$-lactams (seesaw effect) in methicillin-resistant Staphylococcus aureus," Antimicrobial Agents and Chemotherapy, vol. 61, no. 1, Article ID e01634-16, 2016.

[59] J. A. Hindler, A. Wong-Beringer, C. L. Charlton et al., "In vitro activity of daptomycin in combination with $\beta$-lactams, gentamicin, rifampin, and tigecycline against daptomycinnonsusceptible Enterococci," Antimicrobial Agents and Chemotherapy, vol. 59, no. 7, pp. 4279-4288, 2015. 
[60] J. R. Smith, K. E. Barber, A. Raut, and M. J. Rybak, “ $\beta$ Lactams enhance daptomycin activity against vancomycinresistant Enterococcus faecalis and Enterococcus faecium in in vitro pharmacokinetic/pharmacodynamic models," Antimicrobial Agents and Chemotherapy, vol. 59, no. 5, pp. 2842-2848, 2015.

[61] B. J. Werth, G. Sakoulas, W. E. Rose, J. Pogliano, R. Tewhey, and M. J. Rybak, "Ceftaroline increases membrane binding and enhances the activity of daptomycin against daptomycinnonsusceptible vancomycin-intermediate Staphylococcus aureus in a pharmacokinetic/pharmacodynamic model," Antimicrobial Agents and Chemotherapy, vol. 57, no. 1, pp. 66-73, 2013.

[62] I. Shafiq, Z. P. Bulman, S. L. Spitznogle et al., "A combination of ceftaroline and daptomycin has synergistic and bactericidal activity in vitro against daptomycin nonsusceptible methicillinresistant Staphylococcus aureus (MRSA)," Infectious Diseases, vol. 49, no. 5, pp. 410-416, 2017.

[63] J. Yim, J. R. Smith, N. B. Singh et al., "Evaluation of daptomycin combinations with cephalosporins or gentamicin against Streptococcus mitis group strains in an in vitro model of simulated endocardial vegetations (SEVs)," Journal of Antimicrobial Chemotherapy, vol. 72, no. 8, pp. 2290-2296, 2017.

[64] C. Dupieux, S. Trouillet-Assant, C. Camus et al., "Intraosteoblastic activity of daptomycin in combination with oxacillin and ceftaroline against MSSA and MRSA," Journal of Antimicrobial Chemotherapy, vol. 72, no. 12, pp. 3353-3356, 2017.

[65] A. D. Berti, E. Theisen, J.-D. Sauer et al., "Penicillin binding protein 1 is important in the compensatory response of Staphylococcus aureus to daptomycin-induced membrane damage and is a potential target for $\beta$-lactam-daptomycin synergy," Antimicrobial Agents and Chemotherapy, vol. 60, no. 1, pp. 451458, 2016.

[66] J.-H. Jiang and A. Y. Peleg, "Daptomycin-nonsusceptible Staphylococcus aureus: the role of combination therapy with daptomycin and gentamicin," Gene, vol. 6, no. 4, pp. 1256-1267, 2015.

[67] G. Aktas and S. Derbentli, "In vitro activity of daptomycin combined with dalbavancin and linezolid, and dalbavancin with linezolid against MRSA strains," Journal of Antimicrobial Chemotherapy, vol. 72, no. 2, pp. 441-443, 2017.

[68] G. Aktas and S. Derbentli, "In vitro activity of daptomycin combinations with rifampicin, gentamicin, fosfomycin and fusidic acid against MRSA strains," Journal of Global Antimicrobial Resistance, vol. 10, pp. 223-227, 2017.

[69] A. D. H. Snyder, B. J. Werth, P. Nonejuie et al., "Fosfomycin enhances the activity of daptomycin against vancomycinresistant Enterococci in an in vitro pharmacokineticpharmacodynamic model," Antimicrobial Agents and Chemotherapy, vol. 60, no. 10, pp. 5716-5723, 2016.

[70] L. Phee, M. Hornsey, and D. W. Wareham, "In vitro activity of daptomycin in combination with low-dose colistin against a diverse collection of Gram-negative bacterial pathogens," European Journal of Clinical Microbiology \& Infectious Diseases, vol. 32, no. 10, pp. 1291-1294, 2013.

[71] M. Ghosh, P. A. Miller, U. Möllmann et al., "Targeted antibiotic delivery: selective siderophore conjugation with daptomycin confers potent activity against multidrug resistant Acinetobacter baumannii both in vitro and in vivo," Journal of Medicinal Chemistry, vol. 60, no. 11, pp. 4577-4583, 2017.

[72] N. C. Silva, S. Silva, B. Sarmento, and M. Pintado, "Chitosan nanoparticles for daptomycin delivery in ocular treatment of bacterial endophthalmitis," Drug Delivery, vol. 22, no. 7, pp. 885893, 2015.

[73] H. Jiang, M. Xiong, Q. Bi, Y. Wang, and C. Li, "Self-enhanced targeted delivery of a cell wall- and membrane-active antibiotics, daptomycin, against staphylococcal pneumonia," Acta Pharmaceutica Sinica B (APSB), vol. 6, no. 4, pp. 319-328, 2016.

[74] K. Zheng, M. I. Setyawati, T.-P. Lim, D. T. Leong, and J. Xie, "Antimicrobial cluster bombs: silver nanoclusters packed with daptomycin," ACS Nano, vol. 10, no. 8, pp. 7934-7942, 2016.

[75] J. R. Arregui, S. P. Kovvasu, and G. V. Betageri, "Daptomycin proliposomes for oral delivery: formulation, characterization, and in vivo pharmacokinetics," AAPS PharmSciTech, vol. 19, no. 4, pp. 1802-1809, 2018. 

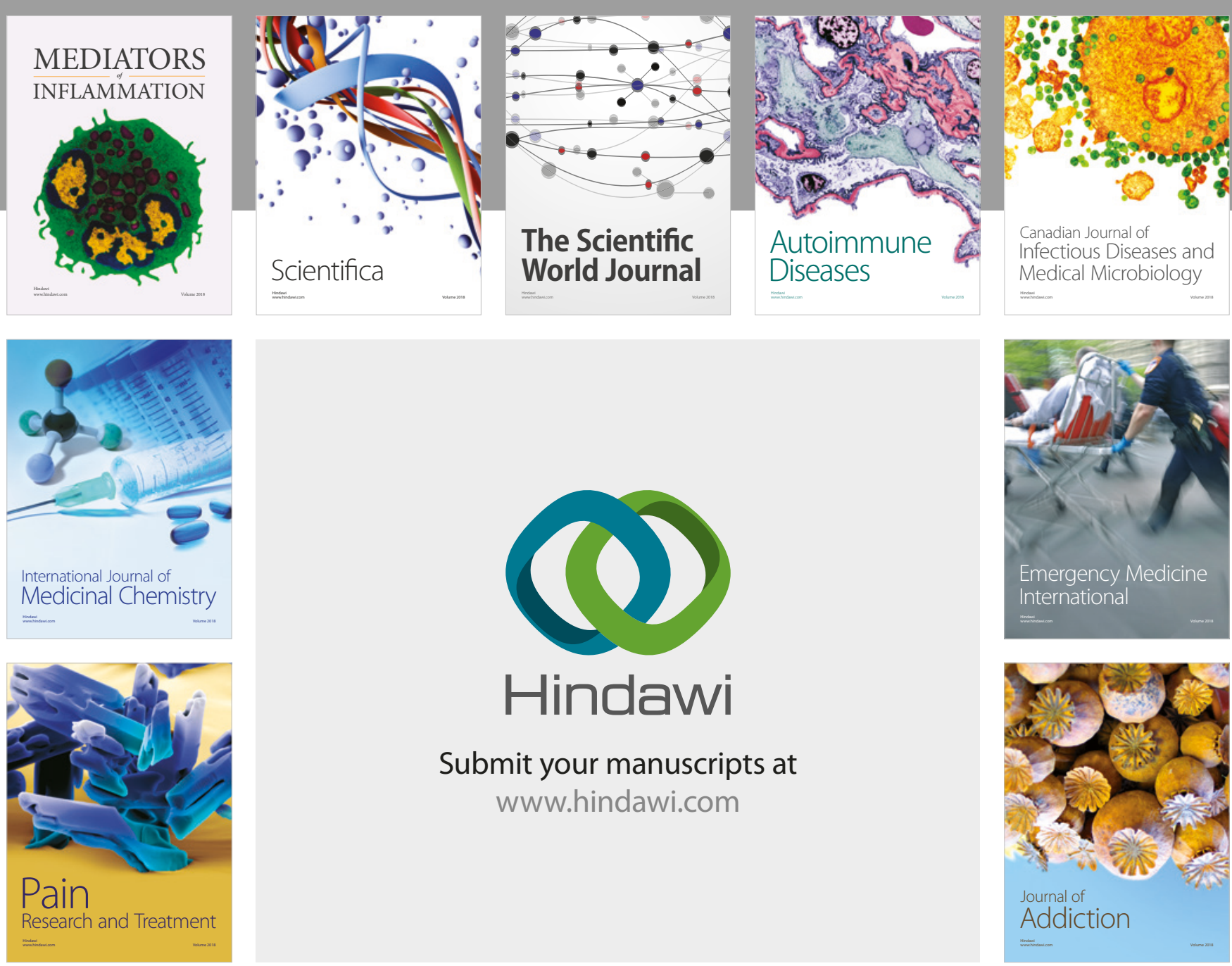

Canadian Journal of
Infectious Diseases and Medical Microbiology

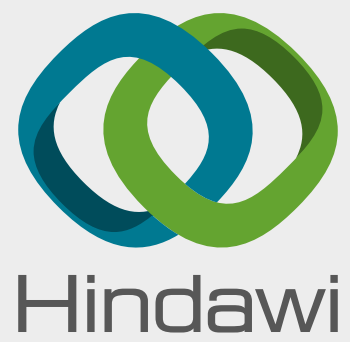

Submit your manuscripts at

www.hindawi.com
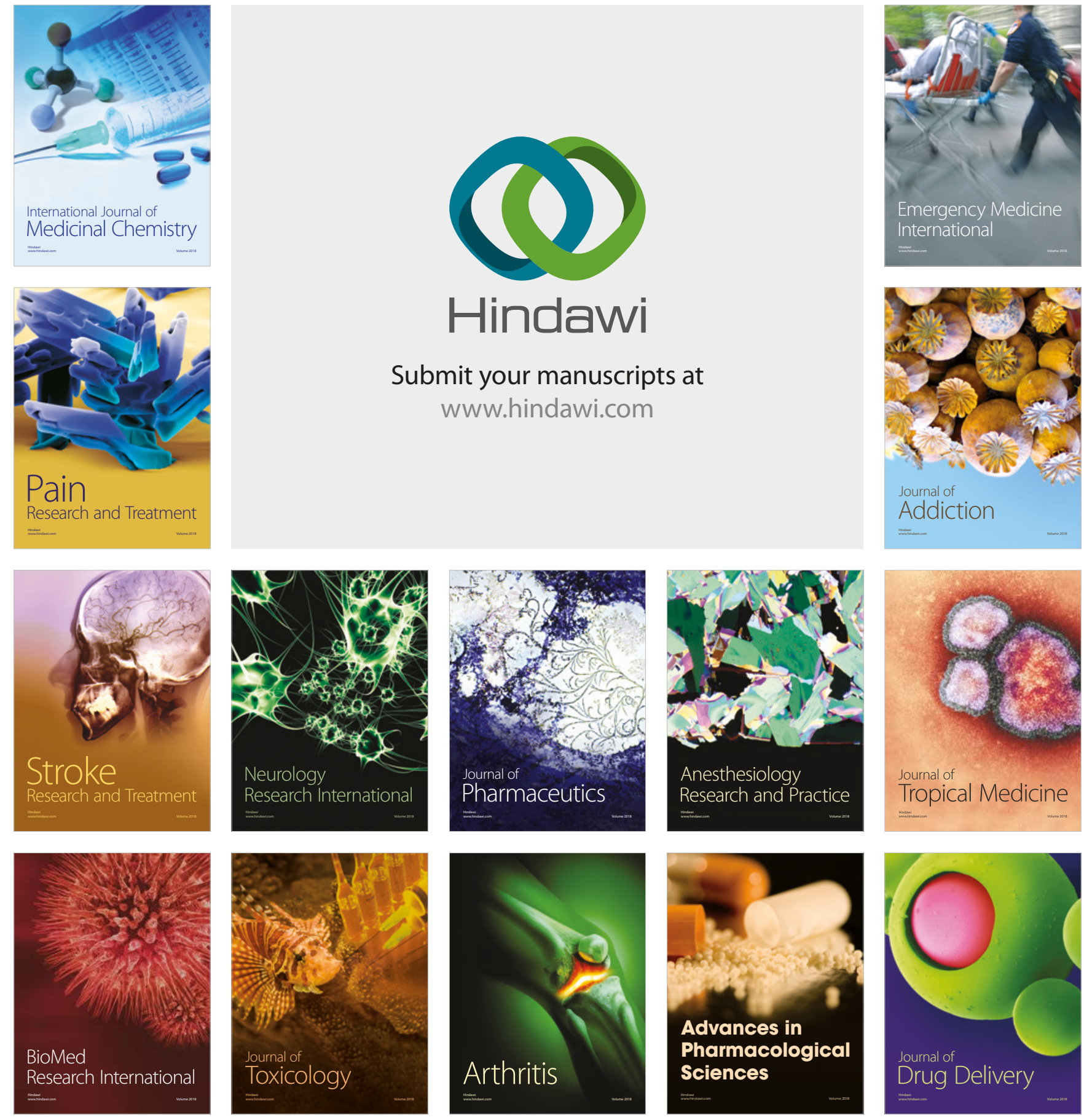